\title{
CONTROL DE PH EN FOTOBIORREACTORES UTILIZANDO UN GPC BASADO EN EVENTOS
}

\author{
Andrzej Pawlowski*, José L. Guzmán*, Manuel Berenguel*, Ignacio Fernández*, \\ Francisco G. Acién ${ }^{\dagger}$, Julio E. Normey-Rico ${ }^{\ddagger}$ \\ * Departamento de Informática \\ Universidad de Alemría \\ 04120 Almería, España \\ ${ }^{\dagger}$ Departamento de Ingeniería Química, \\ Universidad de Almería \\ 04120 Almería, España \\ ${ }_{\ddagger}$ Deptartamento de Automação e Sistemas, \\ Universidade Federal de Santa Catarina \\ Cx.P. 476, 88040-900, Florianópolis, SC, Brasil
}

Emails: a.pawlowski@ual.es, joseluis.guzman@ual.es, beren@ual.es, ifernandez@ual.es, facien@ual.es, julio@das.ufsc.br

\begin{abstract}
This work presents the use of an event-based model predictive control algorithm to regulate the pH in a microalgae production process. The control aim is to maintain the $\mathrm{pH}$ within specific limits and to minimize $\mathrm{CO}_{2}$ losses. The control scheme is based on a Generalized Predictive Control (GPC) algorithm with sensor deadband. This control algorithm allows to establish a tradeoff between control performance and number of actuations, what can be directly related with $\mathrm{CO}_{2}$ losses. The control structure is evaluated through simulations using a nonlinear model for microalgal production in tubular photobioreactors. The obtained results are compared with classical time-based solutions using well-known control performance indexes.
\end{abstract}

Keywords — model predictive control, GPC, event-based control, bio-process, microalgae pH control

Resumen - Este trabajo presenta la aplicación de un control predictivo generalizado basado en eventos para el control de pH de una planta de producción de microalgas. Con la estrategia de control propuesta se consigue alcanzar un compromiso entre prestaciones y número de actuaciones, que están directamente relacionadas con las pérdidas de $\mathrm{CO}_{2}$ del proceso. Los resultados que se obtienen en simulación usando un modelo no lineal de la producción de microalgas en fotobioreactores tubulares se comparan con los que se consiguen con soluciones clásicas de control.

Palavras-chave — control predictivo, GPC, sistemas de control basado en eventos, control de $\mathrm{pH}$

\section{Introducción}

La producción de microalgas utilizando fotobioreactores se está convirtiendo en una práctica común en el sector de producción de biomasa. Los motivos principales de este crecimiento se deben principalmente a su alto rendimiento, alto contenido de aceite en algunas cepas, bajo consumo de agua y a que pueden ser producidas en áreas áridas. Además de esto, al mismo tiempo las microalgas ayudan a fijar el $\mathrm{CO}_{2}$ contribuyendo de esta forma a su mitigación (Acién et al., 2012).

Cuando las cepas se destinan a productos de alto valor agregado, es necesario el uso de fotobiorreactores cerrados, tales como los fotobiorreactores tubulares, que son los utilizados en este trabajo. En ellos es posible controlar las condiciones de operación y evitar la contaminación, cumpliendo con los requisitos para la industria farmacéutica (Wang et al., 2012). La calidad de la biomasa puede ser alcanzada mediante el mantenimiento de las principales variables en un cierto nivel óptimo, especialmente el $\mathrm{pH}$. En fotobiorreactores tubulares, el control del pH es realizado por medio de la inyección de dióxido de carbono puro; aunque también pueden ser utilizados otros gases de combustión. Hay varias cuestiones a tener en cuenta con relación a la inyección de $\mathrm{CO}_{2}$. Por un lado, la concentración de $\mathrm{CO}_{2}$ no debe superar el umbral que produce la inhibición de las microalgas, y por otra parte, nunca puede ser inferior a la mínima concentración que limita el crecimiento (Acién et al., 2012). Además, el suministro de dióxido de carbono puro puede constituir hasta el $30 \%$ del coste total de producción de microalgas (Acién et al., 2012). Por otra parte, las pérdidas de carbono en los fotobiorreactores tubulares puede ser superior al $50 \%$, pero se puede reducir por debajo del $30 \%$ mediante el correcto diseño y operación del fotobiorreactor (Acién et al., 2012). Para reducir éste aún más, es necesario diseñar estrategias de control avanzado que tengan en cuenta el fenómeno de mezclado y de transferencia de masa que se produce en el sistema (García et al., 2003; Sierra et al., 2008). Por lo tanto, el problema de control principal para estos procesos consiste en regular el $\mathrm{pH}$ del sistema en el valor deseado, minimizando al mismo tiempo las pérdidas de $\mathrm{CO}_{2}$. En (Berenguel et al., 2004) y 
(Fernández et al., 2010) se utilizan un control predictivo generalizado (GPC) y un PID con acción anticipativa, respecitvamente, para hacer frente a este problema, usando un modelo lineal simplificado de la relación entre el $\mathrm{CO}_{2}$ inyectado y el $\mathrm{pH}$. En ambos trabajos se mejora el resultado obtenido con el control todo/nada usado en la industria.

Las estrategias de control basadas en eventos son cada vez más populares en la práctica, ya que permiten encontrar una solución de compromiso entre el rendimiento y el esfuerzo de control de una forma muy sencilla (Årzén, 1999; Pawlowski et al., 2012). Así, el control basado en eventos se ajusta a los requisitos deseados para el problema de control de $\mathrm{pH}$ descrito anteriormente. Como es bien conocido, el control basado en eventos se caracteriza por la capacidad de adaptarse de forma que las acciones de control se aplican de manera asíncrona y sólo cuando sea realmente necesario, pudiendo así alcanzar tanto la reducción del esfuerzo de control (minimización de $\mathrm{CO}_{2}$ y por lo tanto sus pérdidas), así como para mantener el $\mathrm{pH}$ en un rango determinado.

En este trabajo, la estrategia de control GPC basada en eventos propuesta en (Pawlowski et al., 2012) se utiliza para controlar el $\mathrm{pH}$ de un fotobiorreactor y alcanzar los requisitos del sistema. El detector de eventos usa la técnica de cruce de nivel y de tiempo límite (Årzén, 1999). Con esta idea, las acciones de control sólo se calculan cuando la salida del proceso se encuentra fuera de una cierta banda alrededor de la consigna. La estrategia de control se prueba mediante simulaciones en un modelo de fotobiorreactor tubular nolineal (Fernández et al., 2012) y los resultados son comparados con un control todo-nada y un GPC tradicional usado en (Berenguel et al., 2004).

\section{Modelos y dinámica del sistema}

El cultivo de microalgas es un sistema de tres fases (sólida, líquida y gaseosa) en que las células son generalmente consideradas como parte de la fracción líquida, donde su tasa de crecimiento es una función de la concentración de nutrientes en el líquido. En este sentido, la disponibilidad de nutrientes por las células es una función del grado de mezcla en la fase líquida de acuerdo con el diseño del reactor. En general, la fase gaseosa está compuesta de $\mathrm{CO}_{2}$ y de aire. Se puede usar dióxido de carbono puro o mezclas de dióxido de carbono con otros gases para suministrar dióxido de carbono al cultivo de microalgas. El dióxido de carbono en fase gaseosa es transportado a la fase líquida para proporcionar carbono inorgánico para el crecimiento y también para el control de pH del cultivo. Este carbono inorgánico es asimilado en las células a una velocidad específica, de acuerdo con la velocidad de fotosíntesis (Acién et al., 2012). En el caso del oxígeno, producido por la fotosíntesis, su transferencia es en el sentido opuesto, desde el estado líquido hacia el gaseoso, acumulándose en el líquido por lo que debe ser eliminado. Por lo tanto, un modelo de crecimiento de microalgas debe considerar la velocidad de fotosíntesis, el estado de la mezcla y la transferencia de masa gas-líquido en el sistema. De acuerdo con estos principios, un modelo general no lineal del crecimiento de microorganismos fotosintéticos en fotobiorreactores fue desarrollado en (Fernández et al., 2012) y se utiliza en este trabajo como la planta virtual en las simulaciones realizadas.

Para el diseño del sistemas de control, se utiliza un modelo simplificado para la variable controlada, en este caso el pH. El pH del cultivo es influenciado principalmente por dos fenómenos: suministro de $\mathrm{CO}_{2}$ y la radiación solar. El suministro de $\mathrm{CO}_{2}$ contribuye a la formación de ácido carbónico provocando una disminución de $\mathrm{pH}$ del cultivo. Por otro lado, las microalgas realizan la fotosíntesis en presencia de radiación solar generando $\mathrm{O}_{2}$ y consumiendo $\mathrm{CO}_{2}$, causando así una aumento gradual en el $\mathrm{pH}$. De esta forma la salida del proceso es el $\mathrm{pH}$ del cultivo, la abertura de la valvula inyectora de $\mathrm{CO}_{2}$ es la variable de control, y la radiación solar es la principal perturbación del sistema. El comportamiento del proceso se puede representar por la siguiente ecuación diferencial simplificada (Berenguel et al., 2004), (Sierra et al., 2008), (Fernández et al., 2010):

$$
\tau \underline{d p H}
$$


$\mathrm{pH}$

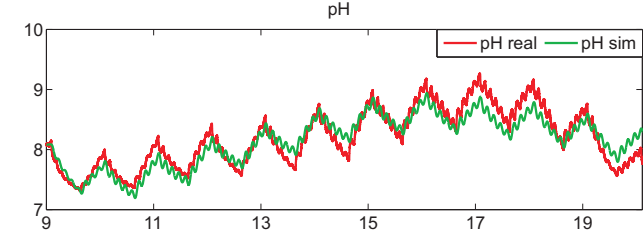

PWM - Añchura del pulso

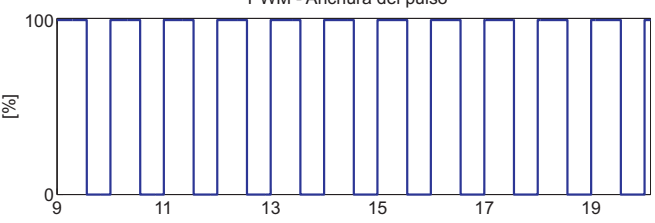

Radiación solar

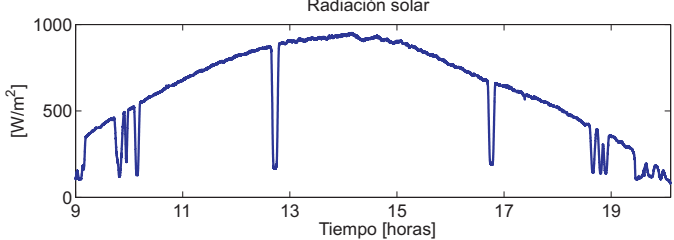

Figura 1: Resultados de la validación del modelo.

orden debido a la recirculación del cultivo a través del receptor solar $T F_{2}$. Por otra parte, se observó un retardo, $t_{r}$, impuesto por el flujo constante de aire de entrada y la distancia entre el punto de inyección de $\mathrm{CO}_{2}$ y el sensor de $\mathrm{pH}$. Finalmente, el efecto de la radiación solar sobre el pH es capturado por un modelo de primer orden, representado por $T F_{3}$. Este modelo lineal ha sido validado correctamente en torno a los puntos de operación deseados, obteniendo $k_{1}=-2.2 \mathrm{pH} \mathrm{V}^{-1}, \tau_{1}=53$ $\min , \omega_{n}=0.014 \mathrm{rad} s^{-1}, \delta=0.042, t_{r}=4.7 \mathrm{~min}$, $k_{r}=0.002 \mathrm{~m}^{2} \mathrm{~W}^{-1}, \mathrm{y} \tau_{r}=182 \mathrm{~min}$. Un ejemplo de validación se puede ver en la figura 1.

\section{Esquema de control GPC basado en eventos}

En esta sección se resume el algoritmo GPC basado en eventos utilizado en este trabajo, que se desarrolló originalmente en (Pawlowski et al., 2012).

\subsection{Estructura general}

De forma general, un controlador basado en eventos consiste en dos partes: un detector de eventos y un controlador (Åström, 2007). El detector de eventos informa al controlador cuándo una nueva acción de control debe ser calculada debido a la ocurrencia de un nuevo evento. En este caso, el controlador está compuesto por un conjunto de

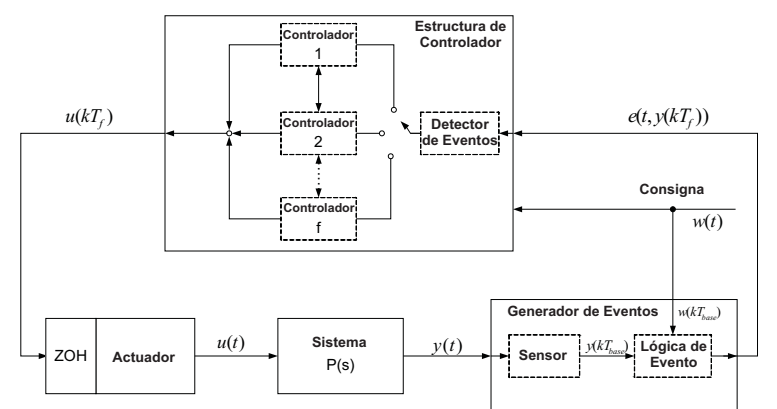

Figura 2: Esquema de control GPC basado en eventos. controladores GPC, de tal manera que uno de ellos se seleccionará de acuerdo con el instante de tiempo cuando se detectó un nuevo evento. La estructura completa de control basado en eventos se muestra en la figura 2, considerando: el sistema, el actuador, el controlador y el generador de eventos. El sistema funciona utilizando la siguiente idea:

- La salida del proceso es muestreada usando un tiempo de muestreo constante, $T_{\text {base }}$, en el bloque de generador de eventos, mientras la acción de control se calcula y se aplica al proceso mediante un tiempo de muestreo variable $T_{f}$.

- $T_{f}$ se define como el múltiplo de $T_{b a s e}\left(T_{f}=\right.$ $\left.f T_{\text {base }}, f \in\left[1, n_{\max }\right]\right)$ y verifica $T_{f} \leq T_{\max }$, siendo $T_{\max }=n_{\max } T_{\text {base }}$ el tiempo de muestreo máximo.

- $T_{\text {base }}$ y $T_{\max }$ se definen teniendo en cuenta el proceso y las especificaciones de lazo cerrado, siguiendo los métodos clásicos para la selección del tiempo de muestreo.

- Tras la aplicación de una acción de control en instante de tiempo $t$, la salida del proceso está monitorizada por el bloque de generador de eventos a cada tiempo de muestreo base, $T_{\text {base }}$. Esta información es utilizada por el bloque detector de eventos, que verifica si la salida del proceso cumple algunas condiciones específicas. Si se cumplen estas condiciones, un evento es generado con un periodo de muestreo $T_{f}$ y se computa una nueva acción de control.

- Nótese que de acuerdo con la descripción anterior, las acciones de control se calcularán basándose en un tiempo de muestreo variable, $T_{f}$. Por esta razón, se utiliza un conjunto de controladores GPC, donde cada controlador GPC está diseñado para un tiempo de muestreo específico $T_{f}$. Por otro lado, es necesario el remuestreo de las señales para evitar cambios indeseables en la acción de control en cada cambio entre los controladores.

\subsection{Algoritmo GPC}

Como se señaló anteriormente, la estructura del control propuesto se basa en el uso del algoritmo GPC. Cada uno de los controladores individuales de este conjunto está implementado como el GPC clásico utilizando el modelo descrito por (2). El controlador GPC consiste en la aplicación de una secuencia de control que minimiza una función de coste de la siguiente forma (Camacho and Bordóns, 2007):

$$
J=\sum_{j=N_{1}^{f}}^{N_{2}^{f}}\left[\hat{y}^{f}(t+j \mid t)-w(t+j)\right]^{2}+\sum_{j=1}^{N_{u}^{f}} \lambda^{f}\left[\Delta u^{f}(t+j-1)\right]^{2}
$$


donde $\hat{y}^{f}(t+j \mid t)$ es la predicción óptima de $j$ muestras hacia adelante de la salida del sistema basada en datos hasta el tiempo $t, \Delta u^{f}(t+j-1)$ son los incrementos futuros de la señal control y $w(t+j)$ es la referencia futura, teniendo en cuenta todas las señales con los tiempos de muestreo $T_{f}\left(t=k T_{f}, k \in Z^{+}\right)$. Por otra parte, el algoritmo cuenta con los siguientes parámetros de sintonía: el horizonte de predicción mínimo y máximo, $N_{1}^{f}$ y $N_{2}^{f}$, el horizonte de control $N_{u}^{f}$, el factor de ponderación del control futuros, $\lambda^{f}$ (Camacho and Bordóns, 2007). El objetivo de GPC consiste en calcular la secuencia de control futura $u^{f}(t), u^{f}(t+1), \ldots, u^{f}\left(t+N_{u}^{f}-1\right)$ de tal manera que las salidas futuras de la planta $y^{f}(t+j)$ alcanzan $w(t+j)$. Esto se realiza minimizando $J$.

\subsection{Muestreo de señal basada en eventos}

En el esquema de la figura 2, se puede observar que el muestreo basado en eventos está gobernado por el bloque de generador de eventos. Este bloque incluye dos diferentes tipos de condiciones con el fin de generar nuevos eventos. Cuando una de estas condiciones se cumple, se genera un nuevo evento, y a continuación, los valores actuales de las variables de proceso se transmiten al bloque controlador para calcular una nueva acción de control (inyecciones de $\mathrm{CO}_{2}$ en este caso). La primera condición comprueba la variable de proceso y se basa en el muestreo por cruce de nivel (Miskowicz, 2006), que en el caso de seguimiento de referencia, tiene la siguiente forma, $|w(t)-y(t)|>\beta$, tratando de detectar si la salida del proceso, $y(t)=p H(t)$, está siguiendo la referencia, $w(t)=w_{p H}(t)$, con la tolerancia especificada $\beta$. La segunda condición está basada en el tiempo, y viene dada por $T_{\max }$ : $t-t_{e_{i}} \geq T_{\max }$, donde $t_{e_{i}}$ es el tiempo cuando se generó el útlimo evento $e_{i}$. Estas condiciones se comprueban con el tiempo de muestreo más rápido $T_{\text {base }}$, que permite generar los eventos con un tiempo de muestreo variable $T_{f}$. Nótese que este periodo de muestreo variable determina el tiempo de muestreo del bucle cerrado que se utiliza para el cálculo de la nueva acción de control.

\subsection{Técnicas de muestreo y remuestro de la señal}

Tal como se describió anteriormente, el cálculo de una nueva acción de control se realiza con un periodo de muestreo variable $T_{f}$. Por lo tanto, con el fin de implementar el algoritmo de control GPC, los valores pasados de las variables del proceso y de las señales de control deben estar disponibles y muestreados con este periodo de muestreo $T_{f}$. Teniendo en cuenta este requisito, es necesario aplicar técnicas de remuestreo para las señales correspondientes.

\subsubsection{Remuestreo de la variable controla- da}

Como se discutió anteriormente, el bloque controlador sólo recibe el nuevo estado de la salida del proceso cuando se genera un nuevo evento. Esta información esta almacenada en el bloque controlador y es remuestreada para generar un vector $y^{b}$ incluyendo los valores pasados de la salida del proceso con muestro $T_{\text {base }}$. El remuestreo de la salida del proceso se lleva a cabo mediante el uso de una aproximación lineal entre dos eventos consecutivos y posteriormente esta aproximación lineal se muestrea con $T_{b a s e}$, creando el vector $y^{b}(k)$ con $k=0, T_{\text {base }}, 2 T_{\text {base }}, 3 T_{\text {base }}, \ldots$ Una vez que la señal de salida del proceso esta remuestreada, la información pasada requerida se obtiene de acuerdo con el nuevo tiempo de muestreo $T_{f}$. Esta operación resulta en una nueva señal, $y^{f}$, con la salida del proceso muestreada con $T_{f}$. Como el resultado de esta operación se obtiene el vector $y^{f}$, que contiene la información pasada del proceso con el nuevo periodo de muestreo $T_{f}$ para utilizarla en los cálculos de la nueva acción de control.

\subsubsection{Reconstrucción de las señales de control pasadas}

El procedimiento es similar al descrito anteriormente para el remuestreo de la salida del proceso. Se dispone de un vector de señal de control, $u^{b}$, que se utiliza siempre para almacenar la señal de control cada $T_{\text {base }}$ muestras. Sin embargo, el procedimiento para la señal de control se hace en el sentido contrario al de la salida del proceso. En primer lugar, se obtiene la información pasada y después se actualiza la señal $u^{b}$, considerando la generación de un nuevo evento, que resulta en un nuevo periodo de muestreo $T_{f}=f T_{\text {base }}$. Ahora, la información pasada para el nuevo periodo de muestreo, $T_{f}$, es calculada a partir de los valores pasados de $u^{b}$ que se almacenan en una variable llamada $u_{p}^{f}$. Posteriormente, esta información, junto con los últimos valores de salida de proceso $y^{f}$, se utilizarán para calcular la nueva acción de control, $u^{f}\left(T_{f}\right)=u^{b}(k)$. Una vez calculada la nueva acción de control, se actualiza $u^{f}\left(T_{f}\right)=u^{b}(k)$ manteniendo constante los valores de señal entre dos eventos consecutivos.

\section{Simulaciones}

Esta sección presenta los resultados de simulación del control basado en eventos aplicado al control de $\mathrm{pH}$ en fotobiorreactores tubulares, donde el modelo no lineal se utiliza como la planta virtual. Con el fin de comparar los resultados obtenidos por la técnica propuesta se utilizan dos estrategias de control, específicamente: controlador todo/nada $(\mathrm{T} / \mathrm{N})$ y controlador GPC clásico. Las simulaciones se llevaron a cabo para un período de 7 días, donde se han considerado diferentes perfiles 
Cuadro 1: Los resultados de simulación y los índices de redimiento 

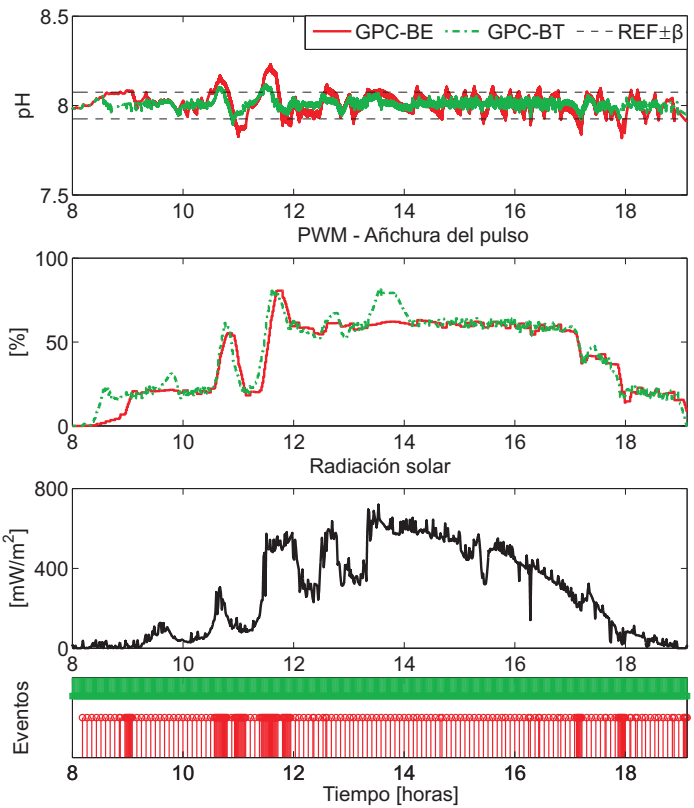

Figura 3: Los resultados de control para un día con radiación solar afectada por nubes.

pérdidas. Sin embargo, el rendimiento del control obtenido es ligeramente peor en comparación con GPC clásico. Por otro lado, es posible obtener una situación de compromiso entre el rendimiento de control y el coste de mantenimiento de la planta.

\section{Conclusiones}

En este trabajo se ha utilizado una estrategia de control GPC basada en eventos para reducir las pérdidas de $\mathrm{CO}_{2}$ en el control del $\mathrm{pH}$ de fotobiorreactores tubulares, manteniendo un nivel de rendimiento de control aceptable. La idea principal consiste en actualizar el proceso controlado sólo cuando se produce una desviación significativa del valor de consigna. El esquema de control presentado proporciona un ahorro en los gastos de mantenimiento de planta, minimizando las pérdidas de $\mathrm{CO}_{2}$. Los beneficios obtenidos se alcanzan a costa de una disminución del rendimiento del control. El compromiso entre el rendimiento del control deseado y las pérdidas de $\mathrm{CO}_{2}$ pueden ser implementadas fácilmente durante la etapa de diseño del sistema de control ajustando el valor de la banda muerta de sensor.

\section{Agradecimientos}

Este trabajo ha sido parcialmente financiado por los siguientes proyectos: PHB2009-0008 (financiado por el Ministerio Español de Educación, CNPq; CAPES-DGU 220/2010); DPI2010-21589-C05-04 y DPI2011-27818-C02-01 (financiados por el Ministerio de Economía y Competitividad de España y los fondos FEDER), y apoyado por la Fundación Cajamar.

\section{Referencias}

Acién, F. G., Fernández, J. M., Magán, J. J. and Molina, E. (2012). Production cost of a real microalgae production plant and strategies to reduce it, Biotechnology Advances 30: 13441353. DOI: 10.1016/j.biotechadv.2012.02.005

Årzén, K. E. (1999). A simple event-based PID controller, Proceedings of the 14th IFAC World Congress . Beijing, China.

Åström, K. J. (2007). Event based control, Analysis and Design of Nonlinear Control Systems pp. 127-147. Springer-Verlag.

Berenguel, M., Rodríguez, F., Acién, F. G. and García, J. L. (2004). Model predictive control of ph in tubular photobioreactors, Journal of Process Control 14: 377-387. DOI: 10.1016/j.jprocont.2003.07.001

Camacho, E. F. and Bordóns, C. (2007). Model Predictive Control, Springer-Verlag, London.

Fernández, I., Acién, F. G., Fernández, J. M., Guzmán, J. L., Magán, J. J. and Berenguel, M. (2012). Dynamic model of microalgal production in tubular photobioreactors, Bioresource Technology 126: 172-181.

Fernández, I., Peña, J., Guzmán, J. L., Berenguel, M. and Acién, F. G. (2010). Modelling and control issues of $\mathrm{pH}$ in tubular photobioreactors, Proceedings of the 11th IFAC Symposium on Computer Applications in Biotechnology. Leuven, Belgium.

García, J. L., Berenguel, M., Rodríguez, F., Fernández, J. M., Brindley, C. and Acién, F. G. (2003). Minimization of carbon losses in pilot-scale outdoor photobioreactors by model-based predictive control, Biotechnology and Bioengineering 84: 533-543. DOI: 10.1002/bit.10819

Miskowicz, M. (2006). Send-On-Delata conept. An event-based data reporting strategy, Sensors 1: $29-63$.

Pawlowski, A., Guzmán, J. L., Normey-Rico, J. E. and Berenguel, M. (2012). A practical approach for generalized predictive control within an event-based framework, Computers and Chemical Engineering 41: 52-66. DOI: 10.1016/j.compchemeng.2012.03.003

Sierra, E., Acién, F. G., Fernández, J., García, J., González, C. and Molina, E. (2008). Characterization of a flat plate photobioreactor for the production of microalgae, Chemical Engineering Journal 138: 136-147. DOI: 10.1016/j.cej.2007.06.004

Wang, B., Lan, C. Q. and Horsman, M. (2012). Closed photobioreactors for production of microalgal biomasses, Biotechnology Advances 30: 904-912. DOI:

10.1016/j.biotechadv.2012.01.019 